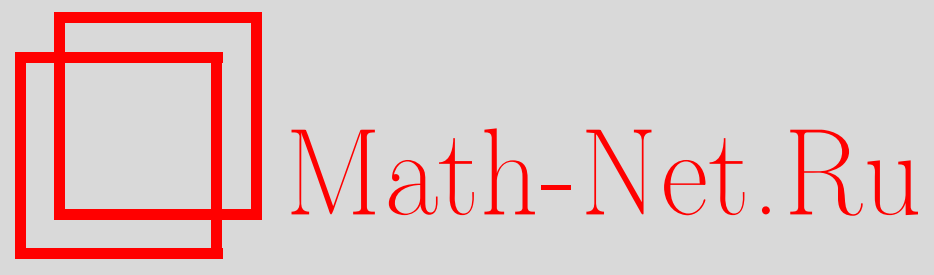

A. Soltani, A. Parvardeh, Simple random measures and simple processes, Теория вероятн. и ее примен., 2005, том 50, выпуск 3, 533-548

DOI: https://doi.org/10.4213/tvp93

Использование Общероссийского математического портала MathNet.Ru подразумевает, что вы прочитали и согласны с пользовательским соглашением

http://www . mathnet.ru/rus/agreement

Параметры загрузки:

IP : 54.164 .48 .24

26 апреля 2023 г., 14:57:50

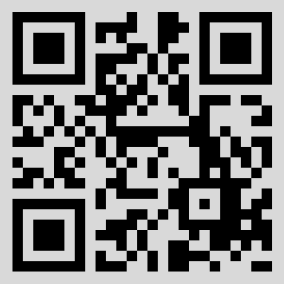




\title{
SIMPLE RANDOM MEASURES AND SIMPLE PROCESSES
}

\begin{abstract}
Простая случайная мера - это конечная сумма случайных мер с непересекающимися носителями. Вводится и изучается класс простых случайных мер, порожденный случайной мерой $\left(\Phi_{1}, \ldots, \Phi_{m}\right)$ и измеримыми отображениями $T_{1}, \ldots, T_{m}$. Это приводит к введению класса процессов, называемых простыми, который включает в себя стационарные процессы и периодически коррелированные процессы с дискретным временем. Наше исследование затрагивает вопросы характеризации и моделирования спектральных и временных областей. Также показана роль спектральных ядер в анализе нестационарных процессов.
\end{abstract}

Ключевъе слова и фразы: случайная мера, простая случайная мера, простые процессы, разложение Холецкого, спектральное ядро, спектральная область, временная область, моделирование.

1. Introduction and preliminaries. Research activities on nonstationary processes are growing. There are many varieties of nonstationarities. In contrast to the stationary processes, there is no global time series analysis for nonstationary processes. For some types of nonstationary processes and their applications see [1], [7], [2] and [11], [9], [10], [12], [5], [4], [3]. In this work we introduce and study a class of processes which are in general nonstationary, and assume spectral kernels of certain types, we call such processes simple processes, that include stationary processes and discrete time periodically correlated (cyclostationary) processes. After short preliminaries given below, in Section 2 we introduce simple random measures and their spectral densities and spectral kernels. Simple processes are introduced in Section 3, where spectral domain and time domain together with the elements of prediction are established. Section 4 is devoted to the simulation of simple processes.

Let $(D, \mathscr{D})$ be a measurable space, and let $\mathscr{L}^{2}(\Omega, \mathscr{F}, \mathbf{P})$ be the Hilbert space of zero mean complex random variables $X$ on the probability space

* Department of Statistics, College of Sciences, Shiraz 71454, Iran; Department of Statistics and Operations Research, Faculty of Sciences, Kuwait University, P. O. Box 5969 Safat-13060, Kuwait; e-mail: soltani@sun01.susc.ac.ir; soltani@kuc01.kuniv.edu.kw

** Department of Statistics, College of Sciences, University of Isfahan, Isfahan; Iran; e-mail: a.parvardeh@stat.ui.ac.ir 
$(\Omega, \mathscr{F}, \mathbf{P})$ with finite second moments, $\mathbf{E}|X|^{2}<\infty$. A mapping $\Phi: \mathscr{D} \rightarrow$ $\mathscr{L}^{2}(\Omega, \mathscr{F}, \mathbf{P})$ is a second order random measure on $\mathscr{D}$ if

1) $\Phi(\varnothing)=0$,

2) $\mathbf{E}\left|\Phi\left(\bigcup_{i=1}^{\infty} A_{i}\right)-\sum_{i=1}^{n} \Phi\left(A_{i}\right)\right|^{2} \longrightarrow 0$ as $n \rightarrow \infty$,

for disjoint sets $A_{1}, A_{2}, \ldots$ in $\mathscr{D}$. The equality $\Phi\left(\bigcup_{i=1}^{\infty} A_{i}\right)=\sum_{i=1}^{\infty} \Phi\left(A_{i}\right)$ is a short notation expressing convergence in the second moment. A random measure $\Phi$ is independently scattered (in short IS), if for every finite sequences of disjoint sets $A_{1}, \ldots, A_{n}$ in $\mathscr{D}, \Phi\left(A_{1}\right), \ldots, \Phi\left(A_{n}\right)$ are independent; $\Phi$ is called orthogonally scattered (in short OS) if $\Phi(A) \perp \Phi(B)$, whenever $A \cap B=\varnothing$.

Let $\Phi$ be an OS random measure, then $\mu(A)=\mathbf{E}|\Phi(A)|^{2}$ defines a positive measure on $(D, \mathscr{D})$ which is called the spectral measure of $\Phi$. A set $B \in \mathscr{D}$ is a support of $\Phi$, if it is a support of $\mu$.

Let $\Phi_{1}, \ldots, \Phi_{d}$ be OS (IS) random measures on $\mathscr{D}$ for which $\left\{\Phi_{1}(A), \ldots, \Phi_{d}(A)\right\}$ and $\left\{\Phi_{1}(B), \ldots, \Phi_{d}(B)\right\}$ are orthogonal (independent) if $A \cap B=\varnothing$; then for every $A, B \in \mathscr{D}, \mathbf{\Phi}=\left(\Phi_{1}, \ldots, \Phi_{d}\right)$ is a $d$-dimensional random measure. The spectral measure of $\boldsymbol{\Phi}$, denoted by $\boldsymbol{\mu}$, is defined by

$$
\boldsymbol{\mu}(A)=\mathbf{E}(\mathbf{\Phi}(A) \overline{\mathbf{\Phi}(A)}), \quad A \in \mathscr{D},
$$

which is a $d \times d$ matrix $\boldsymbol{\mu}=\left[\mu_{k j}\right]$, where, for each $k, j, \mu_{k j}(\cdot)=\mathbf{E} \Phi_{k}(\cdot) \overline{\Phi_{j}(\cdot)}$ and defines a complex measure on $\mathscr{D}$. The spectral domain of $\Phi$ is the space $\mathscr{L}^{2}(\boldsymbol{\mu})$ that consists of all vector-valued $\mathscr{D}$-measurable functions $\mathbf{f}=$ $\left(f_{1}, \ldots, f_{d}\right)$ on $D$ such that

$$
\int_{D} \mathbf{f}(d \boldsymbol{\mu}) \mathbf{f}^{*}<\infty
$$

where ${ }^{*}$ stands for the complex-transpose. The integral given above is defined in [8].

2. Simple random measures. To depart from OS (IS) random measures, it is natural to study random measures that are finite sums of OS (IS) random measures with disjoint supports.

Suppose $\Phi_{1}, \ldots, \Phi_{m}$ are OS (IS) random measures with spectral measures $\mu_{1}, \ldots, \mu_{m}$ respectively, which are defined on $\mathscr{D}$. Assume $\mu_{j}$ is supported by $B_{j}, i=1, \ldots, m$, and $B_{i} \cap B_{j}=\varnothing$ for $i \neq j$. Define

$$
\Phi(A)=\Phi_{1}\left(A \cap B_{1}\right)+\cdots+\Phi_{m}\left(A \cap B_{m}\right),
$$

then $\Phi$ is a random measure on $\mathscr{D}$ which is called here a «simple random measure» and in general is not OS (IS).

For the random measure $\Phi$ in (2.1):

$$
\begin{aligned}
\mathbf{E}|\Phi(d x)|^{2} & =\mu_{j}(d x), \quad x \in B_{j}, \\
\mathbf{E} \Phi(d x) \overline{\Phi(d y)} & =\mathbf{E} \Phi_{j}(d x) \overline{\Phi_{i}(d y)}, \quad x \in B_{j}, \quad y \in B_{i},
\end{aligned}
$$


which exhibits that $\Phi$ is not OS unless $\Phi_{j} \perp \Phi_{i}$ for $j \neq i$, furthermore $\mathbf{E}|\Phi(A)|^{2}$ cannot be specified unless $\nu_{j k}(E, F)=\mathbf{E} \Phi_{j}(E) \overline{\Phi_{k}(F)}$ is known for $E \subset B_{j}, F \subset B_{k}$.

A situation that $\nu_{j k}(E, F)$ can be specified is when $\Phi$ is induced by an $m$-variate random measure and $m$ measurable mappings. To construct such $\Phi$, let $\Psi=\left(\Psi_{1}, \ldots, \Psi_{m}\right)$ be an $m$-variate OS (IS) random measure on $B_{1}$, with spectral measure $\boldsymbol{\mu}=\left[\mu_{j k}\right]$, and let $T_{j}: B_{1} \rightarrow B_{j}, j=1, \ldots, m$, be measurable mappings. Let $\Phi_{j}=\Psi_{j} T_{j}^{-1}$ in (2.1), then for the resulting $\Phi$,

$$
\nu_{j k}(E, F)=\mathbf{E} \Psi_{j}\left(T_{j}^{-1}(E)\right) \overline{\Psi_{k}\left(T_{k}^{-1}(F)\right)}=\mu_{j k}\left(T_{j}^{-1}(E) \cap T_{k}^{-1}(F)\right) .
$$

Interestingly, in this case

$$
\nu(A \times C)=\sum_{j, k} \nu_{j k}\left(A \cap B_{j}, C \cap B_{k}\right)
$$

defines a measure (product measure) on $(D \times D, \mathscr{D} \times \mathscr{D})$ and is called the spectral measure of $\Phi$.

If the mappings $T_{1}, \ldots, T_{m}$ are one-to-one, then on each rectangle $B_{j} \times B_{k}, \nu$ is supported by the curve $y=T_{k} T_{j}^{-1}(x)$, namely,

$$
\nu\left(E_{j k}\right)=\mu_{j k}\left\{T_{j}^{-1}(x):\left(x, T_{k} T_{j}^{-1}(x)\right) \in E_{j k}\right\}, \quad E_{j k} \subset B_{j} \times B_{k} .
$$

Indeed for $E \subset D \times D$ let $E_{j, k}=E \cap\left(B_{j} \times B_{k}\right)$, then

$$
\begin{aligned}
\nu(E) & =\sum_{j, k} \nu\left(E_{j, k}\right)=\sum_{l=-m+1}^{m-1} \sum_{j=\max \{1-l, 1\}}^{\min \{m-l, m\}} \nu\left(E_{j, l+j}\right) \\
& =\sum_{j=1}^{m} \nu\left(E_{j, j}\right)+\sum_{l=1}^{m-1}\left\{\sum_{j=1}^{m-l} \nu\left(E_{j, l+j}\right)+\sum_{j=m-l+1}^{\min \{2 m-l, m\}} \nu\left(E_{j, j+l-m}\right)\right\} .
\end{aligned}
$$

Now define $\mu_{0}, \ldots, \mu_{m-1}$ on $D$ through

$$
\begin{aligned}
& \mu_{0}(A)=\sum_{j=1}^{m} \mu_{j, j}\left(T_{j}^{-1}\left(A \cap B_{j}\right)\right), \\
& \mu_{l}(A)=\sum_{j=m-l+1}^{\min \{2 m-l, m\}} \mu_{j, j+l-m}\left(T_{j}^{-1}\left(A \cap B_{j}\right)\right)+\sum_{j=1}^{m-l} \mu_{j, l+j}\left(T_{j}^{-1}\left(A \cap B_{j}\right)\right),
\end{aligned}
$$

for $l=1, \ldots, m-1$, then

$$
\nu(E)=\sum_{l=0}^{m-1} \mu_{l}\{x \in D:(x, y) \in E, \text { for some } y\}, \quad E \subset D \times D .
$$

Therefore the spectral measure $\nu$ is specified by $m$ complex measures $\mu_{0}, \ldots, \mu_{m-1}$ on $D$, that are determined by $\boldsymbol{\mu}=\left[\mu_{j k}\right]$, the spectral measure of the $m$-variate random vector $\boldsymbol{\Phi}=\left(\Phi_{1}, \ldots, \Phi_{m}\right)$. The spectral measure $\boldsymbol{\mu}$ also can be specified from $\mu_{0}, \ldots, \mu_{m-1}$, namely,

$$
\begin{array}{ll}
\mu_{j k}(F)=\mu_{m+k-j}\left(T_{j}(F)\right), \quad k-j<0, & F \subset B_{1}, \\
\mu_{j k}(F)=\mu_{k-j}\left(T_{j}(F)\right), \quad k-j \geqslant 0, & F \subset B_{1} .
\end{array}
$$


Therefore $\nu$ and $\boldsymbol{\mu}$ uniquely specify each other. We arrive at the following theorem.

Theorem 2.1. Let $\mu_{0}, \ldots, \mu_{m-1}$ be complex measures on the measurable space $(D, \mathscr{D})$, let $\left\{B_{1}, \ldots, B_{m}\right\}$ be a partition for $D$ of elements of $\mathscr{D}$, and let $T_{j}: B_{1} \longrightarrow B_{j}, j=1, \ldots, m$, be one-to-one measurable mappings. Then $\left(\mu_{0}, \ldots, \mu_{m-1}\right)$ is the spectrum of a simple random measure, if $\boldsymbol{\mu}=\left[\mu_{j k}\right]$ which is formed from $\left(\mu_{0}, \ldots, \mu_{m-1}\right)$ through (2.5) is the spectral matrix of an $m$-variate random vector $\boldsymbol{\Psi}=\left(\Psi_{1}, \ldots, \Psi_{m}\right)$ supported by $B_{1}$. (In this case we say that $\Phi$ is induced by $\boldsymbol{\Psi}$ and $\left(T_{1}, \ldots, T_{m}\right)$.)

A simple random measure is not in general OS (IS), but it takes its values in the time domain of an OS (IS) random measure. Such observation is useful to specify the spectral domain for purpose of prediction, see [11]. The following lemma clarifies this point.

Lemma 2.1. Let $\boldsymbol{\Psi}=\left(\Psi_{1}, \ldots, \Psi_{m}\right)$ and let $T_{1}, \ldots, T_{m}$ be as in Theorem 2.1 with spectral measure $\boldsymbol{\mu}=\left[\mu_{j k}\right]$. Suppose there is a positive measure $\mu$ on $B_{1}$ for which $\frac{d \mu}{d \mu}=\left[f_{j k}\right]=\mathbf{f}$ exists, and $\mathbf{f}$ is full rank $\mu$-a.e. Then there is an $O S$ random measure $\Lambda$ on $D$ for which

(i) $\mathbf{E}\left|\Lambda\left(T_{j}(A)\right)\right|^{2}=\mu(A), A \subset B_{1}, j=1, \ldots, m$,

(ii) $\Psi_{j}(d x)=\sum_{k=1}^{m} a_{j k}(x) \Lambda\left(T_{k}(d x)\right), j=1, \ldots, m, x \in B_{1}$,

where $a_{j k}(x), j, k=1, \ldots, m$, are complex functions on $D$ and are formed by a decomposition of $\mathbf{f}$, namely,

$$
\mathbf{f}(x)=\mathbf{A}(x) \mathbf{A}^{*}(x), \quad \mathbf{A}(x)=\left[a_{j k}(x)\right], \quad x \in B_{1}
$$

(where $\mathbf{A}^{*}(x)$ is the conjugate transpose of $\mathbf{A}(x)$ ).

P r o of. To construct $\Lambda$, following [8, p. 40], let $\Lambda_{j}(d x)=$ $\mathbf{b}_{j}(x) \boldsymbol{\Psi}^{\prime}(d x), x \in B_{1}, j=1, \ldots, m$, where $\mathbf{b}_{j}(x)$ is the $j$-th row of the $\mathbf{A}^{-1}$. Then $\boldsymbol{\Lambda}=\left(\Lambda_{1}, \ldots, \Lambda_{m}\right)$ is OS on $B_{1}$. Indeed

$$
\mathbf{E} \Lambda_{j}(d x) \overline{\Lambda_{k}(d x)}=\mathbf{b}_{j} \mathbf{f} \mathbf{b}_{k}^{*}(x) \mu(d x)= \begin{cases}0, & j \neq k, \\ \mu(d x), & j=k .\end{cases}
$$

Define $\Lambda(d x)=\Lambda_{j}\left(T_{j}^{-1}(d x)\right), x \in B_{j}, j=1, \ldots, m$. Then

$$
\mathbf{E}\left|\Lambda\left(T_{j}(A)\right)\right|^{2}=\mathbf{E}\left|\Lambda_{j}\left(T_{j}^{-1}\left(T_{j}(A)\right)\right)\right|^{2}=\mu(A), \quad A \subset B_{1}, \quad j=1, \ldots, m,
$$

giving (i). For (ii), note that $\boldsymbol{\Psi}^{\prime}=\mathbf{A} \mathbf{\Lambda}^{\prime}$. Therefore,

$$
\Psi_{j}(d x)=\sum_{k=1}^{m} a_{j k}(x) \Lambda_{k}(d x)=\sum_{k=1}^{m} a_{j k}(x) \Lambda\left(T_{k}(d x)\right), \quad x \in B_{1} .
$$

The spectral measure of $\Lambda$ on $(D, \mathscr{D})$ is given by

$$
\mu_{\Lambda}(A)=\sum_{j=1}^{m} \mu\left(T_{j}^{-1}\left(A \cap B_{j}\right)\right), \quad A \subset D .
$$

The proof is complete. 
$\mathrm{R}$ e $\mathrm{m}$ a $\mathrm{rk}$ 2.1. If the spectral density matrix $\mathbf{f}$ is not full rank, but has rank $q<m, \mu$-a.e., then it follows from [8, Sec. I.9] that the conclusion in Lemma 2.1 is still valid but the number of sets in the partition and the number of transformations should be reduced to $q$.

To proceed, we assume that the assumptions of Lemma 2.1 are fulfilled, $q=m$.

Theorem 2.2. Let $\Phi$ be a simple random measure with spectrum $\left(\mu_{0}, \ldots, \mu_{m-1}\right)$ given by (2.3), and let $\boldsymbol{\mu}=\left[\mu_{j k}\right]$ given by (2.5) satisfy the assumptions of Lemma 2.1. Then there is an OS random measure $\Lambda$ on $(D, \mathscr{D})$ for which

$$
\Phi(A)=\int_{D} h(x, A) \Lambda(d x), \quad A \subset D
$$

where

$$
h(x, A)=\sum_{k=1}^{m} \sum_{j=1}^{m} a_{j k}\left(T_{k}^{-1}(x)\right) I_{T_{k} T_{j}^{-1}\left(A \cap B_{j}\right)}(x) ;
$$

$a_{j k}(x), j, k=1, \ldots, m$, are as in Lemma 2.1 and are related to the matrix $\mathbf{f}$ via $(2.6)$.

$\mathrm{P}$ r o o f. Apply Lemma 2.1 and note that $\Phi$ is induced by an $m$-variate random vector with spectral matrix $\boldsymbol{\mu}$ :

$$
\begin{aligned}
\Phi(A) & =\sum_{j=1}^{m} \Psi_{j}\left(T_{j}^{-1}\left(A \cap B_{j}\right)\right)=\sum_{j=1}^{m} \sum_{k=1}^{m} \int_{T_{j}^{-1}\left(A \cap B_{j}\right)} a_{j k}(x) \Lambda\left(T_{k}(d x)\right) \\
& =\sum_{k=1}^{m} \sum_{j=1}^{m} \int_{T_{k}\left(T_{j}^{-1}\left(A \cap B_{j}\right)\right)} a_{j k}\left(T_{k}^{-1}(x)\right) \Lambda(d x) \\
& =\int_{D} \sum_{k=1}^{m} \sum_{j=1}^{m} a_{k}\left(T_{k}^{-1}(x)\right) I_{T_{k}\left(T_{j}^{-1}\left(A \cap B_{j}\right)\right)}(x) \Lambda(d x) .
\end{aligned}
$$

The proof is complete.

$\mathrm{R}$ e $\mathrm{m}$ a r k 2.2. Two types of decomposition for the density $\mathbf{f}$ in (2.6) are common. Consider the Cholesky decomposition, in which the matrix $\mathbf{A}$ is triangular and the elements on the main diagonal are positive. In this case define complex functions $a_{k}, k=0, \ldots, m-1$, on $D$ through

$$
a_{j k}(x)= \begin{cases}a_{j-k}\left(T_{j}(x)\right) & \text { if } j \geqslant k \\ 0 & \text { if } j<k\end{cases}
$$

giving that

$$
\mathbf{A}(x)=\left[a_{j-k}\left(T_{j}(x)\right)\right]_{j \geqslant k}, \quad x \in B_{1} .
$$

Note that $a_{k}(x)$ is zero on $\bigcup_{j=1}^{k} B_{j}, k=1, \ldots, m-1, a_{0}(x) \neq 0$ on $D$. In this case

$$
\Psi_{j}(d x)=\sum_{k=0}^{j-1} a_{k}\left(T_{j}(x)\right) \Lambda\left(T_{j-k}(d x)\right), \quad j=1, \ldots, m, \quad x \in B_{1} .
$$


The next case is that $\mathbf{A}$ is the boundary value of an analytic matrix with no zero in the interior of the corresponding region. This type of decomposition plays fundamental role in the extrapolation of multivariate stationary processes, [1], [8].

$\mathrm{R}$ e $\mathrm{m}$ a $\mathrm{r} \mathrm{k}$ 2.3. $h(x, A)$ given in (2.7) can be written as

$$
h(x, A)=\sum_{k=1}^{m} \sum_{j=1}^{m} a_{j k}\left(T_{k}^{-1}(x)\right) I_{\left(A \cap B_{j}\right)}\left(T_{j} T_{k}^{-1}(x)\right) I_{B_{k}}(x) ;
$$

it defines a kernel, for $x \in B_{k}, h(x, \cdot)$ is supported by the atoms $T_{j} T_{k}^{-1}(x)$, $j=1, \ldots, m$, with corresponding masses $a_{j k}\left(T_{j} T_{k}^{-1}(x)\right)$. We refer to $h(\cdot, \cdot)$ as the spectral kernel of the simple random measure $\Phi$.

If $(2.6)$ is the Cholesky decomposition, then the kernel $h(x, A)$ is given by

$$
h(x, A)=\sum_{k=1}^{m} \sum_{j=k}^{m} a_{j-k}\left(T_{j} T_{k}^{-1}(x)\right) I_{\left(A \cap B_{j}\right)}\left(T_{j} T_{k}^{-1}(x)\right) I_{B_{k}}(x),
$$

where for $x \in B_{k}, h(x, \cdot)$ is supported by the atoms $T_{j} T_{k}^{-1}(x), j=k, \ldots, m$, with corresponding masses $a_{j-k}\left(T_{j} T_{k}^{-1}(x)\right)$.

$\mathrm{Ex}$ a $\mathrm{m} \mathrm{pl} \mathrm{e} \mathrm{2.1.} \mathrm{Let} m=2$, then in (2.9),

$$
\begin{aligned}
h(x, A)= & a_{11}(x) I_{A \cap B_{1}}(x)+a_{12}\left(T_{2}^{-1}(x)\right) I_{A \cap B_{1}}\left(T_{2}^{-1}(x)\right) I_{B_{2}}(x) \\
& +a_{21}(x) I_{A \cap B_{2}}\left(T_{2}(x)\right) I_{B_{1}}(x)+a_{22}\left(T_{2}^{-1}(x)\right) I_{A \cap B_{2}}(x) .
\end{aligned}
$$

Therefore for $x \in B_{1}, h(x, \cdot)$ is concentrated at $x, T_{2}(x)$ with masses $a_{11}(x)$ and $a_{21}(x)$, respectively. For $x \in B_{2}, h(x, \cdot)$ is concentrated at $x, T_{2}^{-1}(x)$ with masses $a_{22}\left(T_{2}^{-1}(x)\right), a_{12}\left(T_{2}^{-1}(x)\right)$, respectively. Also note that $(2.10)$ becomes

$$
\begin{aligned}
h(x, A) & =a_{0}(x) I_{A \cap B_{1}}(x)+a_{1}\left(T_{2}(x)\right) I_{A \cap B_{2}}\left(T_{2}(x)\right) I_{B_{1}}(x)+a_{0}(x) I_{A \cap B_{2}}(x) \\
& =a_{0}(x) I_{A}(x)+a_{1}\left(T_{2}(x)\right) I_{A \cap B_{2}}\left(T_{2}(x)\right) I_{B_{1}}(x)
\end{aligned}
$$

which exhibits that for $x \in B_{1}, h(x, \cdot)$ is concentrated at $x, T_{2}(x)$ with masses $a_{0}(x), a_{1}\left(T_{2}(x)\right)$, respectively. For $x \in B_{2}, h(x, \cdot)$ is concentrated at $x$ with mass $a_{0}(x)$.

E x a m p l e 2.2. Let $D=\mathbf{R}, B_{1}=(-\infty, 0), B_{2}=[0, \infty)$, and

$$
\Phi(A)=\Phi_{1}(A \cap(-\infty, 0))+\Phi_{2}(A \cap[0, \infty)) .
$$

Assume $\Phi$ is induced by $\Psi=\left(\Psi_{1}, \Psi_{2}\right)$ and $T_{1}(x)=x, T_{2}(x)=-x$ on $B_{1}$. Then $\left\{\mu_{0}, \mu_{1}\right\}$, where

$$
\begin{aligned}
& \mu_{0}(d x)=\mu_{11}(d x), \quad-\infty<x<0 ; \quad \mu_{0}(d x)=\mu_{22}(d x), \quad 0 \leqslant x<\infty, \\
& \mu_{1}(d x)=\mu_{12}(d x), \quad-\infty<x<0 ; \quad \mu_{1}(d x)=\mu_{21}(d x), \quad 0 \leqslant x<\infty,
\end{aligned}
$$

is the spectral measure of $\Phi$. Using (2.10), we see the spectral measure $\nu$ is supported by the lines $y=x, y=-x, x \in \mathbf{R}$. The kernel $h(x, A)$ in $(2.10)$ 
is given by

$$
h(x, A)=a_{0}(x) I_{A}(x)+a_{1}(-x) I_{A \cap B_{2}}\left(T_{2}(x)\right) I_{B_{1}}(x) .
$$

$\mathrm{Ex}$ a $\mathrm{m} \mathrm{p}$ le 2.3. Let in Example 2.2 $T_{2}(x)=x^{2}$. Then $\nu$ is supported by $y=x$ and $y=x^{2} I_{(-\infty, 0)}(x)-\sqrt{x} I_{[0, \infty)}(x)$. The kernel $h(x, A)$ in $(2.10)$ is given by

$$
h(x, A)=a_{0}(x) I_{A}(x)+a_{1}\left(x^{2}\right) I_{A \cap B_{2}}\left(x^{2}\right) I_{B_{1}}(x)
$$

and in (2.9) is given by

$$
\begin{aligned}
h(x, A)= & a_{11}(x) I_{A \cap B_{1}}(x)+a_{12}(-\sqrt{x}) I_{A \cap B_{1}}(-\sqrt{x}) I_{B_{2}}(x) \\
& +a_{21}(x) I_{A \cap B_{2}}\left(x^{2}\right) I_{B_{1}}(x)+a_{22}(-\sqrt{x}) I_{A \cap B_{2}}(x) .
\end{aligned}
$$

Ex a m p l e 2.4. Let $D=[0,2 \pi], B_{1}=[0, a), B_{2}=(a, 2 \pi]$, and let $\Phi$ be induced by $\boldsymbol{\Psi}=\left(\Psi_{1}, \Psi_{2}\right)$ and $T_{1}(x)=x, T_{2}(x)=\frac{2 \pi-a}{a} x+a$. Using (2.10) we see then that $\nu$ is supported by $y=x$ and

$$
y=\left(\frac{2 \pi-a}{a} x+a\right) I_{[0, a)}(x)+\frac{a}{2 \pi-a}(x-a) I_{(a, 2 \pi]}(x) .
$$

The kernel $h(x, A)$ is given by

$$
\begin{aligned}
h(x, A)= & a_{0}(x) I_{(0, a]}(x) \\
& +a_{1}\left(\frac{2 \pi-a}{a} x+a\right) I_{A \cap(a, 2 \pi]}\left(\frac{2 \pi-a}{a} x+a\right) I_{(0, a]}(x) .
\end{aligned}
$$

An interesting class of random measures whose elements follow the construction given above is the class of periodically correlated, PC, random measures on $([0,2 \pi), \mathscr{B}([0,2 \pi)))$. Let $B_{j}=[2 \pi(j-1) / d, 2 \pi j / d), j=1, \ldots, d$, and let $\Psi=\left(\Psi_{1}, \ldots, \Psi_{d}\right)$ be a $d$-variate random vector on $[0,2 \pi / d)$ with the spectral measure $\boldsymbol{\mu}=\left[\mu_{j k}\right]$. Also let $T_{j}(x)=x+2 \pi(j-1) / d, x \in B_{1}$, $j=1, \ldots, d$. Then the resulting simple random measure $\Phi$ is called PC. Its spectral measure $\nu$ on each rectangle $[2 \pi(j-1) / d, 2 \pi j / d) \times[2 \pi(k-1) / d$, $2 \pi k / d)$ is supported by the lines $y=x+2 \pi(k-j) / d$. Hence on $[0,2 \pi) \times[0,2 \pi)$ the measure $\nu$ is supported by the lines $y=x+2 \pi l / d, l=-d+1, \ldots, d-1$. The measures $\nu$ and $\mu_{0}, \ldots, \mu_{d-1}$ are as follows

$$
\begin{aligned}
\mu_{0}(A)= & \sum_{j=1}^{d} \mu_{j j}\left(A \cap\left[\frac{2 \pi(j-1)}{d}, \frac{2 \pi j}{d}\right)-\frac{2 \pi(j-1)}{d}\right) \\
\mu_{l}(A)= & \sum_{j=d-l+1}^{\min \{2 d-l, d\}} \mu_{j, j+l-d}\left(A \cap\left[\frac{2 \pi(j-1)}{d}, \frac{2 \pi j}{d}\right)-\frac{2 \pi(j-1)}{d}\right) \\
& +\sum_{j=1}^{d-l} \mu_{j, l+j}\left(A \cap\left[\frac{2 \pi(j-1)}{d}, \frac{2 \pi j}{d}\right)-\frac{2 \pi(j-1)}{d}\right),
\end{aligned}
$$


and

$$
\nu(E)=\sum_{l=0}^{d-1} \mu_{l}\{x:(x, y) \in E \text { for some } y\} .
$$

Then (2.5) becomes

$$
\begin{array}{ll}
\mu_{j k}(d x)=\mu_{d+k-j}\left(d x+\frac{2 \pi(j-1)}{d}\right), & k-j<0, \\
\mu_{j k}(d x)=\mu_{k-j}\left(d x+\frac{2 \pi(j-1)}{d}\right), & k-j \geqslant 0 .
\end{array}
$$

Therefore $\nu$ and $\boldsymbol{\mu}$ uniquely specify each other. The following Theorem is immediate from Theorem 2.1.

Theorem 2.3. A complex measure $\nu$ on $[0,2 \pi) \times[0,2 \pi)$ is the spectral measure of a $P C$ random measure $\Phi$ on $[0,2 \pi)$ if it is supported by the lines $y=x+2 \pi l / d, l=-d+1, \ldots, d-1$, and $\boldsymbol{\mu}=\left[\mu_{j k}\right]_{d \times d}$ that is formed from $\nu$ via $(2.12)$ is the spectral measure of a d-variate random measure $\left(\Psi_{1}, \ldots, \Psi_{d}\right)$ on $[0,2 \pi / d)$, where in $(2.12)$

$$
\mu_{l}(A)= \begin{cases}\nu\left\{(x, y): x \in A, y=x+\frac{2 \pi l}{d}\right\}, & A \subset\left[0, \frac{2 \pi(d-l)}{d}\right), \\ \nu\left\{(x, y): x \in A, y=x-\frac{2 \pi(d-l)}{d}\right\}, & A \subset\left[0, \frac{2 \pi(d-l)}{d}\right),\end{cases}
$$

for $l=0, \ldots, d-1$. Moreover

$$
\begin{aligned}
\Phi(A)= & \Psi_{1}\left(A \cap\left[0, \frac{2 \pi}{d}\right)\right)+\Psi_{2}\left(A \cap\left[\frac{2 \pi}{d}, \frac{4 \pi}{d}\right)-\frac{2 \pi}{d}\right) \\
& +\cdots+\Psi_{d}\left(A \cap\left[\frac{2 \pi(d-1)}{d}, 2 \pi\right)-\frac{2 \pi(d-1)}{d}\right) .
\end{aligned}
$$

Conversely, if $\Phi$ is a PC random measure on $[0,2 \pi)$, i.e., (2.6) is satisfied, then its spectral measure $\nu$ is given by (2.3) and (2.4).

By Theorem 2.2, the spectral kernel of a PC random measure $\Phi$ is given by

$$
h(x, A)=\sum_{k=0}^{d-1} a_{k}\left(x+\frac{2 \pi k}{d}\right) I_{A \cap[0,2 \pi(d-k) / d)}(x) ;
$$

where $a_{k}$ 's are complex functions on $[0,2 \pi)$ subject to $a_{k}(x)=0$ for $x \in$ $[0,2 \pi j / d), k=0, \ldots, d-1$, and are uniquely determined by the Cholesky factor of the density $\mathbf{f}=\left[f_{j k}\right]$, i.e.,

$$
\begin{gathered}
f(x)=\mathbf{A}(x) \mathbf{A}^{*}(x), \quad 0 \leqslant x<\frac{2 \pi}{d}, \\
\mathbf{A}(x)=\left[a_{j-k}\left(x+\frac{2 \pi j}{d}\right)\right]_{j \geqslant k},
\end{gathered}
$$


where $\mathbf{f}=\frac{d \mu}{d \mu}=\left[f_{j k}\right]$ and $\mu(A+2 \pi l / d)=\mu(A), A \subset[0,2 \pi / d), l=0, \ldots$, $d-1$.

Note that for each $x \in[0,2 \pi), h(x, \cdot)$ is concentrated on the points $x+2 \pi k / d, k=0, \ldots, d-1$.

$\mathrm{R}$ e $\mathrm{m}$ a $\mathrm{k}$ 2.4. As observed in Lemma 2.1, in the matrix form,

$$
\Psi^{\prime}=\mathbf{A} \Lambda^{\prime}
$$

where $\boldsymbol{\Lambda}=\left(\Lambda_{1}, \ldots, \Lambda_{d}\right)$ is the $d$-variate random vector on $[0,2 \pi / d)$ defined by $\Lambda_{j}(d x)=\Lambda(x+2 \pi(j-1) / d), j=1, \ldots, d$. Therefore

$$
\boldsymbol{\Lambda}^{\prime}(d x)=\mathbf{A}^{-1} \Psi^{\prime}(d x)
$$

if $a_{0}(x) \neq 0 \mu$-a.e.

3. Simple processes. We call a stochastic process $\{X(t), t \in G\}$ simple, if it admits the following spectral representation:

$$
X(t)=\int_{D} f_{t}(x) \Phi(d x), \quad t \in G,
$$

where $\Phi$ is a simple random measure, as it appeared in Theorem 2.2, $G$ is a subset of $\mathbf{R}$ and $\left\{f_{t}, t \in G\right\}$ is a collection of deterministic complexvalued measurable functions on $(D, \mathscr{D})$. In this section we focus on simple harmonizable processes, in discrete or continuous time, for which $G=\mathbf{Z}$ or $\mathbf{R}, D=(0,2 \pi]$ or $\mathbf{R}$, respectively, and $f_{t}(x)=e^{i t x}$. The class of simple harmonizable processes is rather large, it contains stationary and discretetime PC processes and many others. In this section we specify the spectral domain of such processes for the purpose of spectral analysis and prediction.

By using the spectral kernel of $\Phi$ in (2.7), the Kolmogorov isomorphism can be established:

$$
X(t) \longleftrightarrow \int_{D} e^{i t y} h(x, d y)=\sum_{k=1}^{m}\left\{\sum_{j=1}^{m} e^{i t T_{j} T_{k}^{-1}(x)} a_{j k}\left(T_{k}^{-1}(x)\right)\right\} I_{B_{k}}(x) .
$$

Therefore the spectral domain is given by

$$
\mathscr{L}_{X}=\overline{\operatorname{span}}\left\{\sum_{k=1}^{m}\left\{\sum_{j=1}^{m} e^{i t T_{j} T_{k}^{-1}(x)} a_{j k}\left(T_{k}^{-1}(x)\right)\right\} I_{B_{k}}(x), t \in G\right\}
$$

in $\mathscr{L}^{2}(D, \mu)$. Since $B_{k}, k=1, \ldots, m$, are disjoint,

$$
\mathscr{L}_{X}=\bigoplus_{k=1}^{m} \mathscr{M}_{k}
$$

where $\mathscr{M}_{k}=\overline{\operatorname{span}}\left\{\sum_{j=1}^{m} e^{i t T_{j} T_{k}^{-1}(x)} a_{j k}\left(T_{k}^{-1}(x)\right), t \in G\right\}$ in $\mathscr{L}^{2}\left(B_{k}, \mu\right)$. Let $\mathscr{N}_{k}=\overline{\operatorname{span}}\left\{\sum_{j=1}^{m} e^{i t T_{j}(x)} a_{j k}(x), t \in G\right\}$ in $\mathscr{L}^{2}\left(B_{1}, \mu\right)$, then because of the property of $\mu$ in Lemma 2.1, it follows that

$$
g \in \mathscr{N}_{k} \Longleftrightarrow g \circ T_{k}^{-1} \in \mathscr{M}_{k} .
$$


As a $g \in \mathscr{N}_{k}$ is the $\mathscr{L}^{2}(D, \mu)$-limit of a sequence $\left\{\sum_{j=1}^{m} c_{n}\left(T_{j}(x)\right) a_{j k}(x)\right\}$, where $c_{n}(z)=\sum_{l=1}^{n} a_{n l} e^{i t_{l} z}$, by using an argument very similar to one given in the proof of Lemma 2.9 in [11], $\mathscr{N}_{l}$ can be characterized as

$$
\left\{\sum_{j=1}^{m} c\left(T_{j}(x)\right) a_{j k}(x), \int_{B_{1}}\left|\sum_{j=1}^{m} c\left(T_{j}(x)\right) a_{j k}(x)\right|^{2} d \mu(x)<\infty, t \in G\right\} .
$$

Let use denote this subspace of $\mathscr{L}^{2}(D, \mu)$ by $\mathscr{L}_{\mathrm{d}}^{2}\left(B_{1}, T_{1}, \ldots, T_{m}, a_{1 k}, \ldots\right.$, $\left.a_{m k}, \mu\right)$, the index $\mathrm{d}$ is to emphasize that the factor function $c$ within each term does not depend on $j$. Thus if $g \in \mathscr{L}_{\mathrm{d}}^{2}\left(B_{1}, T_{1}, \ldots, T_{m}, a_{1 k}, \ldots, a_{m k}, \mu\right)$, then $g$ is in $\mathscr{L}^{2}\left(B_{1}, \mu\right)$, it is of the form $g(x)=\sum_{j=1}^{m} c\left(T_{j}(x)\right) a_{j k}(x)$ and is specified by the functions $T_{j}, a_{j k}, j=1, \ldots, m$, and a function $c$. Therefore

$$
\mathscr{L}_{X}=\left.\bigoplus_{k=1}^{m} \mathscr{L}_{\mathrm{d}}^{2}\left(B_{1}, T_{1}, \ldots, T_{m}, a_{1 k}, \ldots, a_{m k}, \mu\right)\right|_{T_{k}^{-1}(x)} I_{B_{k}}(x),
$$

in the sense that if $g \in \mathscr{L}_{X}$, then

$$
g(x)=\sum_{k=1}^{m} g_{k}\left(T_{k}^{-1}(x)\right) I_{B_{k}}(x)
$$

where $g_{k} \in \mathscr{L}_{\mathrm{d}}^{2}\left(B_{1}, T_{1}, \ldots, T_{m}, a_{1 k}, \ldots, a_{m k}, \mu\right)$. It is possible to construct an analogue of (3.2) in the time domain. Let $\mathscr{H}_{X}=\overline{\operatorname{span}}\{X(t), t \in G\}$ represents the time domain of a simple harmonizable process $\{X(t), t \in G\}$. Using the kernel $h$ and [11, Theorem 2.5 (iii)], one can writte $X(t)$ as

$$
\begin{aligned}
X(t) & =\int_{D} \int_{D} e^{i t y} h(x, d y) \Lambda(d x) \\
& =\int_{D} \sum_{k=1}^{m} \sum_{j=1}^{m} e^{i t T_{j} T_{k}^{-1}(x)} a_{j k}\left(T_{k}^{-1}(x)\right) I_{B_{k}}(x) \Lambda(d x) \\
& =\sum_{k=1}^{m} \int_{B_{k}} \sum_{j=1}^{m} e^{i t T_{j} T_{k}^{-1}(x)} a_{j k}\left(T_{k}^{-1}(x)\right) \Lambda(d x) .
\end{aligned}
$$

Now let

$$
X_{k}(t)=\int_{B_{1}} \sum_{j=1}^{m} e^{i t T_{j}(x)} a_{j k}(x) \Lambda\left(T_{k}(d x)\right) .
$$

Note that the processes $X_{k}(t), k=1, \ldots, m$, are pairwise orthogonal and therefore

$$
X(t)=\bigoplus_{k=1}^{m} X_{k}(t), \quad t \in G
$$

giving that

$$
\mathscr{H}_{X}=\bigoplus_{k=1}^{m} \mathscr{H}_{X_{k}}
$$


Also for each $t$

$$
\begin{gathered}
\mathscr{L}_{X, t}=\left.\bigoplus_{k=1}^{m} \mathscr{L}_{\mathrm{d}, t}^{2}\left(B_{1}, T_{1}, \ldots, T_{m}, a_{1 k}, \ldots, a_{m k}, \mu\right)\right|_{T_{k}^{-1}(x)} I_{B_{k}}(x), \\
\mathscr{H}_{X, t}=\bigoplus_{k=1}^{m} \mathscr{H}_{X_{k}, t},
\end{gathered}
$$

where the index $t$ stands for the span closure of the appropriate terms corresponding to all $s \leqslant t$. The formulas (3.5) and (3.6) provide a framework for extrapolation in the spectral domain and the time domain. The best linear predictor of $X(t+\tau)$ based on $\{X(s), s \leqslant t\}$ is denoted by $\widehat{X}_{k}(t, \tau)$ and is given by

$$
\widehat{X}(t, \tau)=\sum_{k=1}^{m} \widehat{X}_{k}(t, \tau),
$$

where

$$
\widehat{X}_{k}(t, \tau)=P_{\mathscr{H}_{X_{k}, t}}\left(X_{k}(t+\tau)\right)
$$

and $P_{\mathscr{H}_{X_{k}, t}}$ stands for the projection on $\mathscr{H}_{X_{k}, t}$. In the spectral domain the predictor is given by

$$
g(t, \tau)=\sum_{k=1}^{m} g_{k}(t, \tau),
$$

where $g_{k}(t, \tau)$ is the projection of $\sum_{j=k}^{m} e^{i(t+\tau) T_{j}(z)} a_{j k}(z)$ onto

$$
\mathscr{L}_{\mathrm{d}, t}^{2}\left(B_{1}, T_{1}, \ldots, T_{m}, a_{1 k}, \ldots, a_{m k}, \mu\right)
$$

evaluated at $T_{k}^{-1}(x)$, for $x \in B_{k}$. The error term is given by

$$
e(t, \tau)=\sum_{k=1}^{m} e_{k}(t, \tau),
$$

where $e_{k}(t, \tau)$ is the length of the corresponding co-projection, $k=1, \ldots, m$.

The covariance function of $\{X(t), t \in G\}$, can be evaluated by either using the measure $\nu$ or using (3.3). Using $\nu$,

$$
\begin{aligned}
R(t, s) & =\int_{D} \int_{D} e^{i t x-i s y} \nu(d x, d y)=\sum_{j=1}^{m} \sum_{l=1}^{m} \int_{B_{1}} e^{i t T_{l}(x)-i s T_{j}(x)} \mu_{j l}(d x) \\
& =\sum_{j=1}^{m} \sum_{l=1}^{m} \int_{B_{1}} e^{i t T_{l}(x)-i s T_{j}(x)} f_{j l} \mu(d x),
\end{aligned}
$$

while using (3.3),

$$
\begin{aligned}
R(t, s) & =\sum_{k=1}^{m} R_{k}(t, s)=\sum_{k=1}^{m} \int_{B_{1}} \sum_{j=1}^{m} \sum_{l=1}^{m} e^{i t T_{j}(x)} a_{j k}(x) e^{-i s T_{l}(x)} \overline{a_{l k}(x)} \mu(d x) \\
& =\sum_{j=1}^{m} \sum_{l=1}^{m} \sum_{k=1}^{m} \int_{B_{1}} e^{i t T_{j}(x)-i s T_{l}(x)} a_{j l}(x) \overline{a_{k l}(x)} \mu(d x) .
\end{aligned}
$$


The decomposition (2.6) ensures that the expressions given above for $\mathbf{R}(s, t)$ are the same.

To derive a moving average process for $\{X(t), t \in G\}$, note that

$$
X_{k}(t)=\int_{B_{1}} g_{t, k}(x) \Lambda\left(T_{k}(x)\right)
$$

where

$$
g_{t, k}(x)=\sum_{j=1}^{m} e^{i t T_{j}(x)} a_{j k}(x) .
$$

Interestingly since $T_{k}$ is one-to-one, $\Lambda \circ T_{k}$ is OS and its spectral measure is $\mu$, as by Lemma 2.1 (i)

$$
\begin{aligned}
\mathbf{E} \Lambda\left(T_{k}(A)\right) \overline{\Lambda\left(T_{k}(B)\right)} & =\mu_{\Lambda}\left(T_{k}(A) \cap T_{k}(B)\right)=\mu_{\Lambda}\left(T_{k}(A \cap B)\right)=\mu(A \cap B) \\
\mathbf{E}\left|\Lambda\left(T_{k}(A)\right)\right|^{2} & =\mu_{\Lambda}\left(T_{k}(A)\right)=\mu(A) .
\end{aligned}
$$

Thus for $\mu=\lambda$, the Lebegue measure,

$$
X_{k}(t)=\int_{B_{1}} g_{t, k}(x) W_{k}(d x), \quad k=1, \ldots, m,
$$

where $W_{k}, l=1, \ldots, m$, are orthogonal Wiener measures. Thus by using Parseval type formula for discrete $t$, we obtain

$$
X_{k}(t)=\sum_{l=-\infty}^{\infty}\left(\widehat{g_{t, k}}\right)(l) Z_{k, l}
$$

where $\left(\widehat{g_{t, k}}\right)(l)$ are Fourier coefficients of the periodic function $g_{t, l}$ in (3.7) supported by the bounded set $B_{1}$, and $\left\{Z_{k, l}\right\}_{l}, k=1, \ldots, m$, are orthogonal white noise series. Whether the representation in (3.8) is nonanticipating depends on the type of the factorization in (2.6) and $T_{1}, \ldots, T_{m}$, resulting in $g_{t, k}$ in (3.7) to become analytic. In this case (3.9) becomes useful for the extrapolation.

4. Simulation. The representation (3.3) together with (3.9) can be used for the simulation of discrete harmonizable simple processes. Note that the type of factorization in (2.6) and whether (3.9) is nonanticipating do not matter for the purpose of simulation. So it is recommended to choose the one that is more convenient. The simulation procedure consists of following steps.

S t e p 1. Decomposing the density $f$, forming the function $g_{t, k}$ in (3.7), and evaluating their Fourier coefficients.

$\mathrm{S}$ t e p 2. Simulating $m$ independent classes of white noise series $\left\{Z_{k, l}\right\}_{l=-\infty}^{\infty}, k=1, \ldots, m$.

$\mathrm{S}$ t e p 3. Apply (3.3) and (3.9) to produce data points for the pro(ess $X(t)$. 
$\mathrm{E} \times \mathrm{x} \mathrm{m} \mathrm{p} \mathrm{l}$ e 4.1. In Example 2.4 consider the following spectral density for $\Psi$ :

$$
\mathbf{f}(x)=\left[\begin{array}{cc}
\frac{\sigma^{2}}{2 \pi} & \frac{-\phi \sigma^{2}}{2 \pi} e^{i 2 \pi x / a} \\
\frac{-\phi \sigma^{2}}{2 \pi} e^{-i 2 \pi x / a} & \frac{\sigma^{2}\left(1+\phi^{2}\right)}{2 \pi}
\end{array}\right], \quad x \in(0, a) .
$$

The Cholesky factorization $\mathbf{f}=\mathbf{A} \mathbf{A}^{*}$ provides that

$$
\mathbf{A}(x)=\left[\begin{array}{cc}
\frac{\sigma}{\sqrt{2 \pi}} & 0 \\
\frac{-\phi \sigma}{2 \pi} e^{-i 2 \pi x / a} & \frac{\sigma}{\sqrt{2 \pi}}
\end{array}\right],
$$

therefore $a_{0}(x)=\sigma / \sqrt{2 \pi}, x \in[0,2 \pi)$, and

$$
a_{1}\left(\frac{2 \pi-a}{a} x+a\right)=\frac{-\phi \sigma}{\sqrt{2 \pi}} e^{-i 2 \pi x / a}, \quad x \in[0, a),
$$

consequently

$$
\begin{aligned}
& g_{t, 1}(x)=e^{i t a} a_{0}(x)+\exp \left\{i\left(\frac{2 \pi-a}{a} x+a\right)\right\} a_{1}\left(\frac{2 \pi-a}{a} x+a\right), \\
& g_{t, 2}(x)=\exp \left\{i\left(\frac{2 \pi-a}{a} x+a\right)\right\} a_{0}\left(\frac{2 \pi-a}{a} x+a\right)
\end{aligned}
$$

giving that, for integer $l$,

$$
\begin{aligned}
\widehat{g}_{t, 1}(l)= & \frac{\sigma}{\sqrt{2 \pi a}} \begin{cases}a & \text { if } \quad l=\frac{-a t}{2 \pi}, \\
\frac{a\left(e^{i t a}-1\right)}{i(2 \pi l+a t)} \quad \text { if } \quad l \neq \frac{-a t}{2 \pi} ;\end{cases} \\
& -\frac{\sigma \phi e^{i t a}}{\sqrt{2 \pi a}} \begin{cases}a & \text { if } l=\frac{2 \pi+a t-2 \pi t}{2 \pi}, 2 \pi t \\
\frac{a\left(e^{-i t a}-1\right)}{i(2 \pi l+2 \pi t-a t-2 \pi)} \quad \text { if } \quad l \neq \frac{2 \pi+a t-2 \pi t}{2 \pi}\end{cases} \\
\widehat{g}_{t, 2}(l)= & \frac{\sigma e^{i t a}}{\sqrt{2 \pi a}} \begin{cases}a & \text { if } \quad l=\frac{a t-2 \pi t}{2 \pi}, \\
\frac{a\left(e^{-i t a}-1\right)}{i(2 \pi l+2 \pi t-a t)} & \text { if } \quad l \neq \frac{a t-2 \pi t}{2 \pi} .\end{cases}
\end{aligned}
$$

Real and imaginary parts of the process for $a=\pi$ (PC process) and $a=1$ are illustrated in Figures 1-4.

5. Acknowledgment. The authors are grateful to a referee for providing valuable comments. 


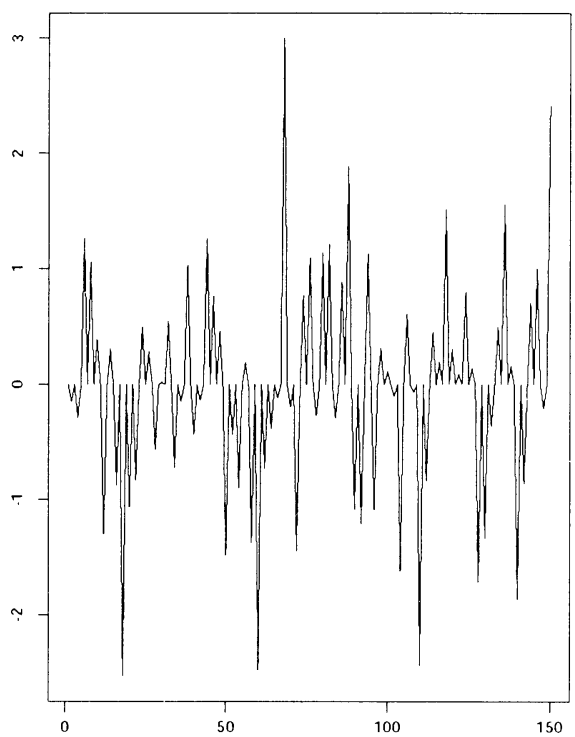

Fig. 1. Simulated series of the real part, Example 4.1, $a=\pi$

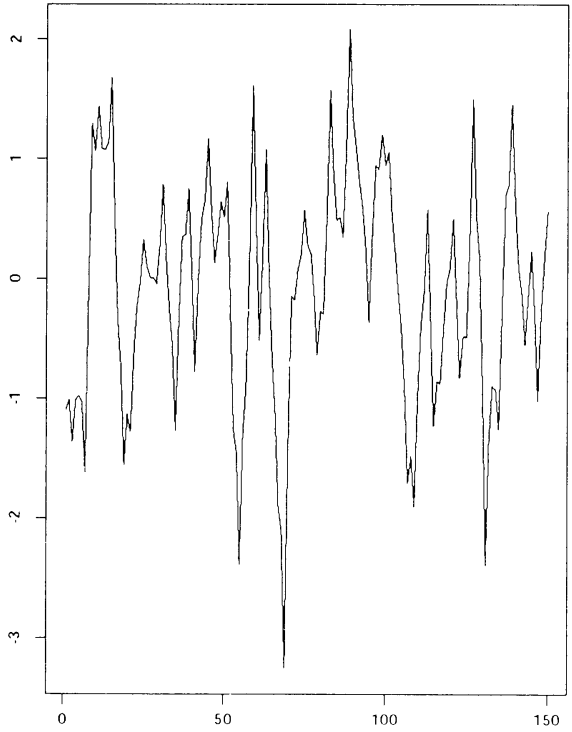

Fig. 2. Simulated series of the imaginary part, Example 4.1, $a=\pi$ 


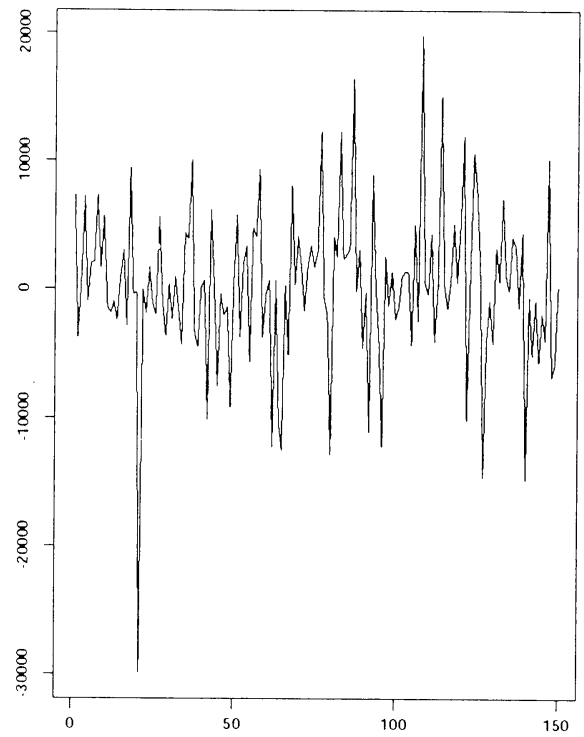

Fig. 3. Simulated series of the real part, Example 4.1, $a=1$

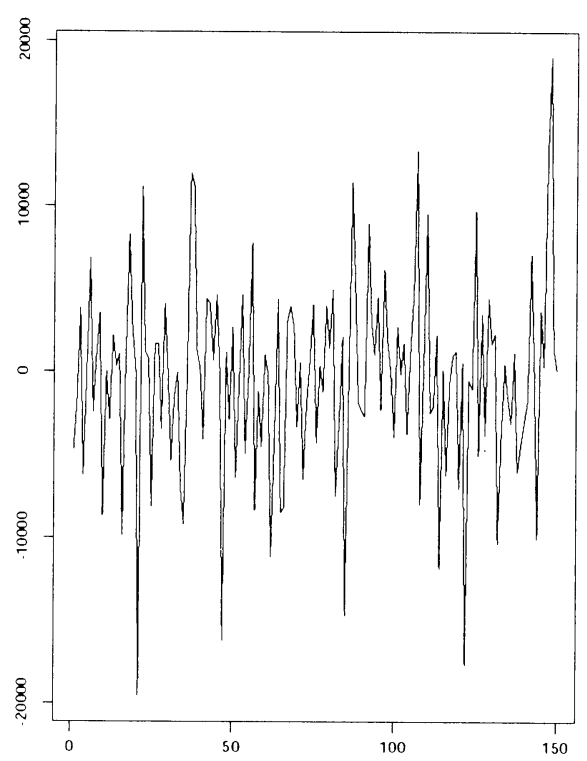

Fig. 4. Simulated series of the imaginary part, Example 4.1, $a=1$ 


\section{REFERENCES}

1. Brockwell P. J., Davis R. A. Time Series: Theory and Methods. New York: SpringerVerlag, 1991, $577 \mathrm{p}$.

2. Gardner W. A. Cyclostationarity in Communications and Signal Processing. New York: IEEE Press, 1994.

3. Гладыщев E. Г. О периодически коррелированных случайных последовательностях. - Докл. АН СССР, 1961, т. 137, № 5, с. 1026-1029.

4. Miamee A. G., Soltani A.R. On spectral analysis of periodically correlated processes. Technical Report. Hampton University, 1994.

5. Nematollahi A.R., Soltani A.R. Discrete time periodically correlated Markov processes. - Probab. Math. Statist., 2000, v. 20, № 1, p. 127-140.

6. Priestley M. B. Evolutionary spectra and nonstationary processes. - J. Roy. Statist. Soc. Ser. B, 1965, v. 27, p. 204-237.

7. Priestley M. B. Spectral Analysis and Time Series. London-New York: Academic Press, 1989, $890 \mathrm{p}$.

8. Розанов Ю. А. Стационарные случайные процессы. М.: Наука, 1963, 284 с.

9. Soltani A. R., Shishebor $Z$. A spectral representation for weakly periodic sequences of bounded linear transformations. - Acta Math. Hungar., 1998, v. 80, № 3, p. 265-270.

10. Soltani A.R., Shishebor Z. Weakly periodic sequences of bounded linear transformations: A spectral characterization. - Georgian Math. J., 1999, v. 6, № 1, p. 91-98.

11. Soltani A.R. A characterization theorem for stable random measures. - Stochastic Anal. Appl., 2000, v. 18, № 2, p. 299-308.

12. Subba Rao T. The fitting of non-stationary time-series models with time-dependent parameter. - J. Roy. Statist. Soc. Ser. B, 1970, v. 32, p. 312-322.

Поступила в редакцию 23.IX.2000

Исправленный вариант 14.VII. 2003 